\title{
Body fluid compartment changes following cardiopulmonary bypass in dogs
}

\author{
Peter B. Manning, Ross A. Morgan, Arnold G. Coran, Theodore Z. Polley, Jr., Robert A. Drongowski, \\ John T. Buckley, and Douglas M. Behrendt
}

Sections of Pediatric Surgery and Thoracic Surgery, The University of Michigan Medical School, Ann Arbor, MI 48109, USA

\begin{abstract}
To investigate whether pulsatile perfusion affects postoperative edema, we examined weight, total body water (TBW), and extracellular fluid (ECF) following cardiopulmonary bypass (CPB) in dogs using three different perfusion systems. Fourteen dogs were divided into three groups differing only in the perfusion system used: Sarns nonpulsatile and pulsatile roller pumps and a University of Texas pulsatile pump. Weight, TBW, and ECF were measured preoperatively and on postoperative days (POD) 1, 3, and 7. No significant differences were seen in body weight, TBW, or ECF between groups. Body weight varied within $10 \%$ of preoperative values. TBW (percent of body weight) rose significantly $(P=0.005)$ to $72 \%$ on POD 3 and 7 . ECF (percent of body weight) rose to $58 \%$ by POD $7(P=0.008)$. These three perfusion systems produced no differences in the pattern of postoperative body fluid distribution after CPB, suggesting that there is no advantage to pulsatile perfusion for the purpose of decreasing postoperative edema.
\end{abstract}

Key words: Body fluid compartments - Cardiopulmonary bypass - Perfusion systems

\section{Introduction}

Postoperative edema following cardiopulmonary bypass (CPB) can cause cardiac and/or pulmonary dysfunction, which in turn contributes significantly to the morbitity of cardiac surgery.

Offprint requests to: A. G. Coran, F7516 Mott Children's Hospital, Ann Arbor, MI 48109, USA
Small children may be particularly susceptible to these complications. Elevation of measured extracellular fluid (ECF) has been repeatedly demonstrated following standard nonpulsatile CPB [2, $5,13]$. Considerable controversy exists, however, regarding the effect of pulsatile versus nonpulsatile perfusion on a number of physiologic changes accompanying CPB [9]. Indirect evidence suggests that more edema results following nonpulsatile bypass, but little specific information is available demonstrating the dependence of body fluid shifts on the type of perfusion system employed. To investigate this relationship we measured changes in weight, total body water (TBW), and ECF following CPB in young dogs using three different perfusion systems.

\section{Methods}

Fourteen beagle dogs ( 6 weeks old, av wt $10 \mathrm{~kg}$ ) were divided into three groups differing only in the perfusion system used: group I-Sarns nonpulsatile roller pump; group II-Sarns pulsatile roller pump; group III-University of Texas externallyvalved, piston-compressed, conduit pulsatile pump. Other components of the circuit included a Bentley neonatal bubble oxygenator and a heat exchanger. The circuit was primed with a blood-crystalloid mixture (approx. hematocrit $=20 \%$ ) with $\mathrm{pH}$ corrected to 7.4 prior to initiation of bypass. Perioperative gentamicin was administered and pentobarbital anesthesia was used for all procedures. Animal welfare policies and standards of the American Association for the Accreditation of Laboratory Animal Care were followed in conjunction with the animal experimentation. Animals were intubated and allowed to breathe spontaneously until the chest was opened. Femoral artery and vein cannulation was performed for monitoring, blood sampling, and fluid infusion. Venoarterial bypass was instituted following heparinization $(200 \mathrm{U} / \mathrm{kg})$ by performing arterial cannulation via the right common carotid artery with the catheter tip placed in the aortic arch, confirmed at autopsy, and venous cannulation via the right atrium using a small right thoracotomy. Near total venous diversion was 


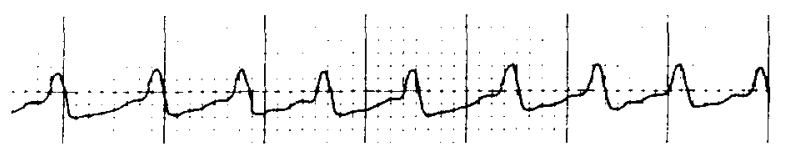

Normal Femoral Pulse

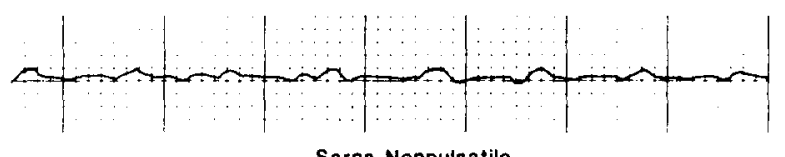

Sarns Nonpulsatile

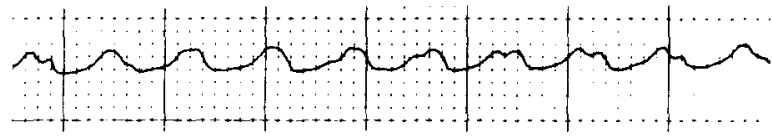

Sarns Pulsatile

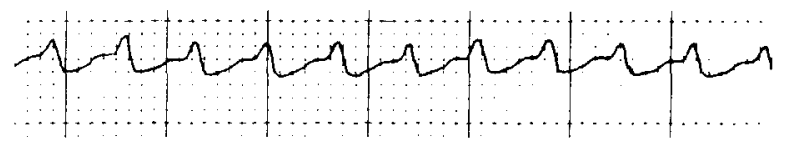

University of Texas Pulsatile

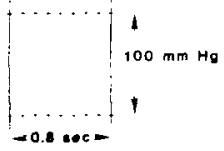

Fig. 1. Femoral pulse wave forms for normal dog, Sarns nonpulsatile pump, Sarns pulsatile pump, and University of Texas pulsatile pump

confirmed by visually identifying an empty right atrium and by inspection of the femoral pulse contour prior to institution of pulsatile bypass, when used.

Following the initiation of stable CPB the animals were cooled to $28^{\circ} \mathrm{C}$ and maintained at that temperature for $90 \mathrm{~min}$, rewarmed to $39^{\circ} \mathrm{C}$, than decannulated. Circuit flow was maintained at $100 \mathrm{ml} / \mathrm{kg}$ per minute during warm perfusion with a mean arterial pressure of $60-70 \mathrm{mmHg}$. For the pulsatile flow groups, pulse pressure was kept between 20 and $30 \mathrm{mmHg}$ (Fig. 1). Sodium bicarbonate was given, if needed, to maintain a pH between 7.3 and 7.4. Postoperatively the animals were allowed food and water ad lib. Weight, TBW, and ECF were

TOTAL. BODY WATER

AND EXTHACELLULLAR FLUID SPACE VS TIME

$\Delta$ Sarns Nonpulsatile o Sarns Pulsatile • UT Pulsatile

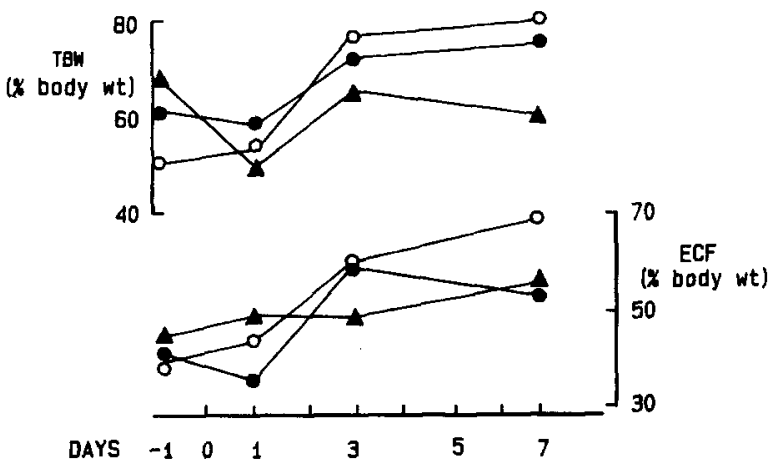

Fig. 2. Comparison of body fluid space changes with three different perfusion systems
TOTAL BODY WATER

AND EXTRACELLULLAR FLUID SPACE AND WEIGHT VS TIME
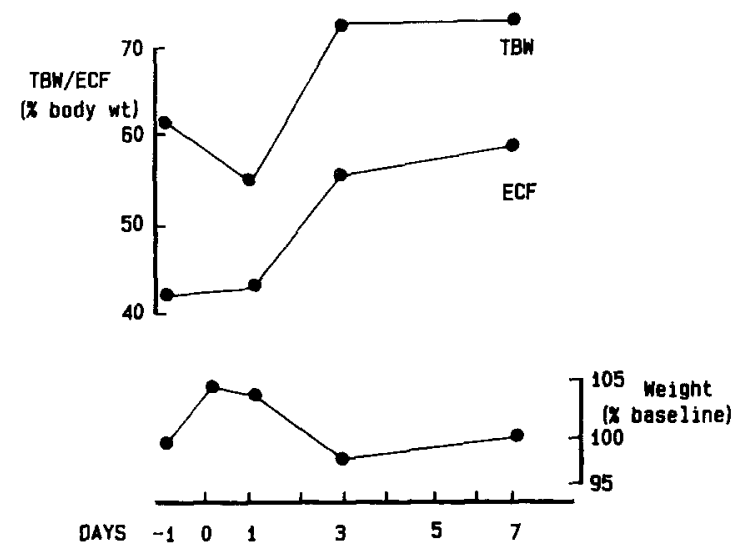

Fig. 3. Body fluid space and weight changes for all animals combined $(n=14)$

measured preoperatively and on postoperative days (POD) 1, 3, and 7. TBW and ECF were determined by measuring the distribution of deuterium oxide [14] and sodium bromide [7], respectively, allowing a 3 -h equilibration period following isotope injection. Data was analyzed using Student's $t$-test with $P<0.05$ considered significant.

\section{Results}

The trends in TBW and ECF changes in each group are depicted in Fig. 2. While both fluid compartments were seen to enlarge in all groups postoperatively, no changes reached statistical significance. Likewise, no significant differences could be demonstrated in weight, TBW, or ECF between the groups. Combination of the data for all 14 animals (Fig. 3 ) shows that body weight varied within $10 \%$ of preoperative values with a peak on POD 1, a low on POD 3, and a return to preoperative levels by POD 7. TBW, expressed as percent of body weight, fell from $61 \%$ to $55 \%$ from preoperatively to POD 1 , then rose to $72 \%$ on POD 3 and POD 7. This postoperative rise was significant $(P=0.005)$. The ECF space rose from $42 \%$ of body weight preoperatively to $58 \%$ by POD 7. This rise was also significant $(P=0.008)$.

\section{Discussion}

In this model of CPB in young dogs, designed to resemble a typical operative course of a child undergoing cardiac surgery, no advantage to pulsatile perfusion could be demonstrated for the purpose of decreasing postoperative ECF expansion. This correlated with the lack of evident differences in the modest amout of peripheral edema noted clinically in each group. A postoperative increase of both TBW and ECF was demonstrated 
for the group as a whole but did not reach statistical significance within each subgroup, possibly due to small sample sizes. Earlier studies in which these fluid spaces were directly measured following standard, nonpulsatile CPB have demonstrated a consistent rise in ECF postoperatively, as seen here, but a less definable change in TBW $[2,5,13]$. These shifts of body fluid have been shown to be most pronounced in patients with a history of preoperative congestive heart failure or when hemodilution is used [6]. One study, looking only at functional changes and myocardial associated with $\mathrm{CPB}$, showed that newborns were much more susceptible to these changes [12]. In many centers hemodilution is avoided in small children because of resultant problems with postoperative edema [3].

In most studies comparing pulsatile and nonpulsatile perfusion, indirect evidence has supported the conclusion that more edema occurs following nonpulsatile perfusion. This evidence takes the form of increased fluid requirements, higher transfusion volumes, lower urine output and creatinine clearance, and greater evident peripheral edema, all seen in the nonpulsatile groups [8-10]. Other studies, however, have failed to show significant differences between the two types of perfusion in terms of fluid balance, particularly when high-flow perfusion was employed $[4,11]$.

These findings are summarized in an excellent review by Hickey et al. [9]. Elsewhere in this review Hickey emphasizes that because of the lack of definition and documentation of the nature of the perfusion used in different studies, meaningful comparisons are difficult to make. Therefore, considerable controversy continues regarding the differences between pulsatile and nonpulsatile perfusion. Bartlett [1] points out that many of the demonstrated differences between these two types of perfusion are related to the flow rate used. No significant difference is noted when flows close to the expected normal cardiac output are employed; however, at flow rates closer to $50 \%$ of expected cardiac output some advantage is demonstrated with pulsatile perfusion.

The aim of this study was to directly measure the changes in TBW and ECF following pulsatile and nonpulsatile perfusion in a model resembling a child undergoing cardiac surgery. Under the conditions employed in this study, which in- cluded flows near expected cardiac output and modest hemodilution, we found no advantage to pulsatile perfusion for the purpose of decreasing edema following cardiopulmonary bypass.

\section{References}

1. Bartlett RH (1984) Hemodynamics and extracorporeal circulation. In: Hagl S, Klovekorn WP, Mayr N, Sebening $F$ (eds) Thirty years of extracorporeal circulation, 1953-1983. Deutsches Herzzentrum, München

2. Beall AC Jr, Johnson PC, Shirkey AL, Crosthwait RW, Cooley DA, DeBakey ME (1964) Effects of temporary cardiopulmonary bypass on extracellular fluid volume and total body water in man. Circulation 29: 59-62

3. Behrendt DM, Austen WG (1985) Patient care in cardiac surgery, 4th edn. Little, Brown \& Co., Boston

4. Boucher JK, Rudy LW, Edmunds LH Jr (1974) Organ blood flow during pulsatile cardiopulmonary bypass. J Appl Physiol 36: 86-90

5. Cleland J, Pluthm JR, Tauxe WN, Kirklin JW (1966) Blood volume and body fluid compartment changes soon after closed and open intracardiac surgery. J Thorac Cardiovasc Surg 52: 698-705

6. Cohn LH, Angell WW, Shumway NE (1971) Body fluid shifts after cardiopulmonary bypass. Effects of congestive heart failure and hemodilution. $J$ Thorac Cardiovasc Surg 62: $423-430$

7. Drongowski RA, Coran AG, Wesley JR (1982) Modification of the serum bromide assay for the measurement of extracellular fluid volume in small subjects. J Surg Res 33: $423-426$

8. Dunn J, Kirsh MM, Harness J, Carroll M, Straker J, Sloan $\mathrm{H}$ (1974) Hemodynamic, metabolic, and hematologic effects of pulsatile cardiopulmonary bypass. $J$ Thorac Cardiovasc Surg 68: 138-147

9. Hickey PR, Buckley MJ, Philbin DM (1983) Pulsatile and nonpulsatile cardiopulmonary bypass: review of a counterproductive controversy. Ann Thorac Surg 36: 720-737

10. Jacobs LA, Klopp EH, Seamone W, Topaz SR, Gott VL (1969) Improved organ function during cardiac bypass with a roller pump modified to deliver pulsatile flow. $J$ Thorax Cardiovasc Surg 58: 703-712

11. Lindberg H, Svennevig J-L, Lilleaasen P, Vatne K. (1984) Pulsatile vs non-pulsatile flow during cardiopulmonary bypass. Comparison of early postoperative changes. Scand J Thor Cardiovasc Surg 18: 195-201

12. Mavroudis C, Ebert PA (1978) Hemodilution causes decreased compliance in puppies. Circulation 58: 155-159

13. Pacifico AD, Digerness S, Kirklin JW (1970) Acute alterations of body composition after open intracardiac operations. Circulation $41: 331-341$

14. Rhodin AGJ, Coran AG, Weintraub WW, Wesley JR (1979) Total body water changes during high volume peripheral hyperalimentation. Surg Gynecol Obstet 148: 196-200

Accepted January 25, 1988 